\title{
METODOLOGÍA PARA LA TRANSICIÓN A UN MODELO DE DIRECCIÓN ESTRATÉGICA SUSTENTADA EN PROCESOS
}

\author{
METHODOLOGY FOR THE TRANSITION TO A MODEL OF STRATEGIC \\ MANAGEMENT BASED ON PROCESSES
}

\author{
Lindsay Rojas Roque ${ }^{1, \downarrow}$, Javier Rivero Villar ${ }^{1}$, Nivian Montes de Oca Martínez
}

\begin{abstract}
RESUMEN
La gestión por procesos gana cada vez más prestigio, entre otras razones por el hecho de que el funcionamiento de la Organización puede proyectarse, dirigirse y mejorarse como un conjunto de procesos interrelacionados para el cumplimiento de los objetivos estratégicos y para la satisfacción de las necesidades, lo que ha determinado la necesidad de búsqueda y desarrollo de nuevos modelos de gestión que permitan la supervivencia y estabilidad de las organizaciones tomando como base la Dirección Estratégica. En la literatura consultada se pudo corroborar la inexistencia de una metodología que integre coherentemente la Dirección Estratégica, la gestión por procesos y la administración de riesgos; es por esto que este artículo tuvo como objetivo diseñar una metodología para la transición a un modelo de Dirección Estratégica sustentada en procesos teniendo en cuenta estos precedentes. La metodología consta de cuatro fases: preparación para la transición, diagnóstico organizacional, diseño estratégico básico, e implementación, seguimiento y control. Se validó en una entidad de Ciencia, Tecnología e Innovación ubicada en la provincia de Mayabeque, Cuba, se definieron las principales brechas, se elaboró el nuevo diseño estratégico, y se propusieron los indicadores estratégicos para facilitar el diseño del Cuadro de Mando Integral de la Organización. La metodología propuesta favorece la flexibilidad en las organizaciones, permitiendo mayor coherencia para realizar los cambios, mejorar el desempeño de la gestión, mayor anticipación frente a desafíos y oportunidades que se generan de las condiciones externas y de la realidad interna, para lograr mayor competitividad y sostenibilidad.
\end{abstract}

Palabras Clave: Modelos de gestión, Planificación Estratégica, Gestión por procesos, Riesgos

\footnotetext{
${ }^{1}$ Centro Nacional de Sanidad Agropecuaria, Carretera Tapaste y Autopista Nacional, San José de las Lajas, Mayabeque, Cuba. orcid.org/0000-0001-6591-0628
}

•Autor de correspondencia: lindsay@censa.edu.cu 
Process management is gaining more and more prestige, among other reasons due to the fact that the functioning of the Organization can be projected, directed and improved as a set of interrelated processes for the fulfillment of the strategic objectives and for the satisfaction of the needs, what has determined the need to search and develop new management models that allow the survival and stability of organizations based on the Strategic Management. In the literature consulted, it was possible to corroborate the lack of a methodology that coherently integrates the Strategic Management, process management, and risk management; this is why this article aimed to design a methodology for the transition to a model of Strategic Management based on processes taking into account these precedents. The methodology consists of four phases: preparation for the transition, organizational diagnosis, basic strategic design and implementation, monitoring and control. It was validated in an entity of Science, Technology, and Innovation located in the province of Mayabeque, Cuba, the main gaps were defined, the new strategic design was elaborated, and strategic indicators were proposed to facilitate the design of the Organization's Balanced Scorecard. The proposed methodology favors flexibility in organizations, allowing greater coherence to make changes, improve management performance, greater anticipation in the face of challenges and opportunities that are generated from external conditions and internal reality, to achieve greater competitiveness and sustainability.

Keywords: Management models, Strategic Planning, Processes, Process management, Risks.

\section{INTRODUCCIÓN}

Las organizaciones en su objetivo de lograr mayor sostenibilidad han aprendido a gestionar o dirigir, anticipándose con decisiones transformadoras que permitan su supervivencia y desarrollo, en medio de un entorno cambiante, hacia una concepción estratégica de la dirección (Alfonso, 2007).

La veracidad de este planteamiento se aprecia al analizar la evolución de los diferentes enfoques de dirección que se han venido introduciendo en las organizaciones en busca del cambio, el crecimiento y el perfeccionamiento. Algunos de estos enfoques son: la dirección por objetivos, la Dirección Estratégica, la Dirección Estratégica basada en valores y más recientemente la Dirección Estratégica integrada (Bolaño et al., 2014).

La Dirección Estratégica Integrada se centra en alcanzar la integración del sistema de gestión de modo que sus elementos o subsistemas estén todos diseñados para asegurar que las prácticas de gestión aplicadas produzcan las habilidades y faciliten las relaciones que le permitan a la Organización alcanzar su visión del futuro en virtud de las estrategias planificadas (Alfonso et al., 2011).

Este nuevo enfoque de dirección se incluye armónicamente con los enfoques de dirección que le preceden, e incorpora en su funcionamiento otros sistemas de gestión como la gestión por procesos, la gestión por competencias, entre otros (Carballal, 2011).

La gestión por procesos gana cada vez más prestigio, entre otras razones, por el hecho de que las Organizaciones son tan eficientes como lo son sus procesos (Zaratiegui, 1999). Una 
medida de su desarrollo evolutivo lo constituye el hecho de que en principio se consideraba sólo como un enfoque o herramienta supeditada o enmarcada dentro de otros sistemas de gestión, sin embargo, actualmente su relevancia y carácter generalizador hacen que poco a poco adquiera personalidad propia (Hernández et al., 2013).

Según Alfonso (2007) el funcionamiento de la Organización puede proyectarse, dirigirse y mejorarse como un conjunto de procesos interrelacionados para el cumplimiento de los objetivos estratégicos y para la satisfacción de las necesidades; pese a esto, en la actualidad las Organizaciones cubanas tienen una tendencia a regirse por un enfoque jerárquico funcional, lo que evidencia la centralización en las tareas y no en los resultados, donde no se observan los vínculos entre las actividades (Alfonso et al., 2011; Álvarez \& Vizcaíno, 2015).

Se está reconociendo la necesidad de que los sistemas modernos de dirección y gestión empresarial desarrollen la administración de riesgos, como una parte integral del proceso de administración, para proyectar acciones que permitan dar una mayor seguridad a la implementación de las estrategias (Cienfuegos, 2009; Ornés, 2011; Bolaño et al., 2011; Lyon \& Hollcroft, 2012; López et al., 2013; Bolaño et al., 2014).

Únase a esto la aprobación e implementación de la ISO 9001 del 2015 que demanda de la Alta Dirección un mayor compromiso, liderazgo y participación, comenzando por el establecimiento de la Dirección Estratégica y siguiendo por la toma de conciencia que debe tenerse acerca del contexto donde opera la Organización y la complejidad de sus relaciones; así como la determinación de los riesgos que pueden afectar su capacidad para cumplir con los objetivos organizacionales establecidos.

En los momentos actuales se requiere de proyecciones estratégicas que posibiliten a los sucesos futuros que preparen el entorno y que sirvan de base para el establecimiento de objetivos, aspecto que se refleja a través de los modelos de Dirección Estratégica. Durante los últimos años ha ganado fuerza la concepción de poner en primer plano los procesos, lo cual no significa que de forma absoluta todos los trabajos y áreas de una organización obligatoriamente deban organizarse sobre esta base, pero las experiencias acumuladas y la realidad de la vida en las organizaciones que han adoptado los sistemas o formas que hacen énfasis en los procesos así lo demuestran (Carballal, 2011).

Tomando como punto de partida estos antecedentes se llevó a cabo un estudio de la literatura tanto nacional como internacional de metodologías para la transición a una gestión por procesos. En el contexto actual cubano destacan el Modelo de Dirección Estratégica Integrada del Sistema de Dirección de la Empresa (DEISDE) (Alfonso, 2007) y el Modelo de Dirección Estratégica basado en la Administración de Riesgos (DE - ARISDE) (Bolaño et al., 2014); a partir del análisis bibliográfico realizado, se pudo corroborar que no existe propiamente una metodología para llevar a cabo la transición de un modelo de Dirección por Objetivos a una Dirección sustentada en procesos, incluyendo la gestión de los riesgos en Organizaciones que basen su sistema de gestión en un modelo de Dirección Estratégica.

Analizados los puntos antes expuestos se plantea como problema la inexistencia de una metodología que establezca los pasos para la transición a un modelo de Dirección Estratégica sustentada en procesos. Por lo que se definen como objetivos de este artículo: (1) diseñar una metodología para la transición a un modelo de Dirección Estratégica sustentada en procesos y (2) validar la metodología propuesta en una entidad de Ciencia, Tecnología e Innovación ubicada en la provincia de Mayabeque, Cuba. Basado en el problema planteado se propone como hipótesis que si se desarrolla coherentemente una metodología que integre coherentemente la Dirección Estratégica, la gestión por procesos y la administración de 
riesgos se favorece la flexibilidad en las organizaciones, permitiendo mayor coherencia para realizar los cambios, mejorar el desempeño de la gestión, mayor anticipación frente a desafíos y oportunidades que se generan de las condiciones externas y de la realidad interna, para lograr mayor competitividad y sostenibilidad.

\section{MATERIALES Y MÉTODOS:}

\section{Bases teórico-metodológicas:}

Se realizó un análisis bibliográfico considerando como palabras clave los términos: Dirección Estratégica, Dirección Estratégica Integrada, Planificación Estratégica, Gestión por procesos, transición a la gestión por procesos, y administración de riesgos. Se consultaron varias fuentes bibliográficas empleando como motores de búsqueda fundamentales Google Scholar y CrossRef,

Se identificaron seis metodologías que incluían elementos de la Dirección Estratégica integrada con énfasis en la dirección por procesos (Negrín, 2003; Junta de Castilla y León, 2004; Román, 2006; Alfonso, 2007; Carballal, 2011; Cejas, 2012), las cuales facilitan el tránsito de las organizaciones hacia una gestión por procesos. La Figura 1 muestra el nivel de inclusión de cada elemento en las metodologías analizadas, y la Figura 2 muestra el porciento de elementos que se incluyen en cada una de las metodologías.

Se observa que hay una alta coincidencia de los elementos que las componen, destacan el procedimiento propuesto por Alfonso (2007) con énfasis especial en el diseño estratégico, y el de Carballal (2011), siendo esta última una de las que más elementos aporta al ser más integradora y flexible en su diseño y aplicación a otras organizaciones que no son empresas.

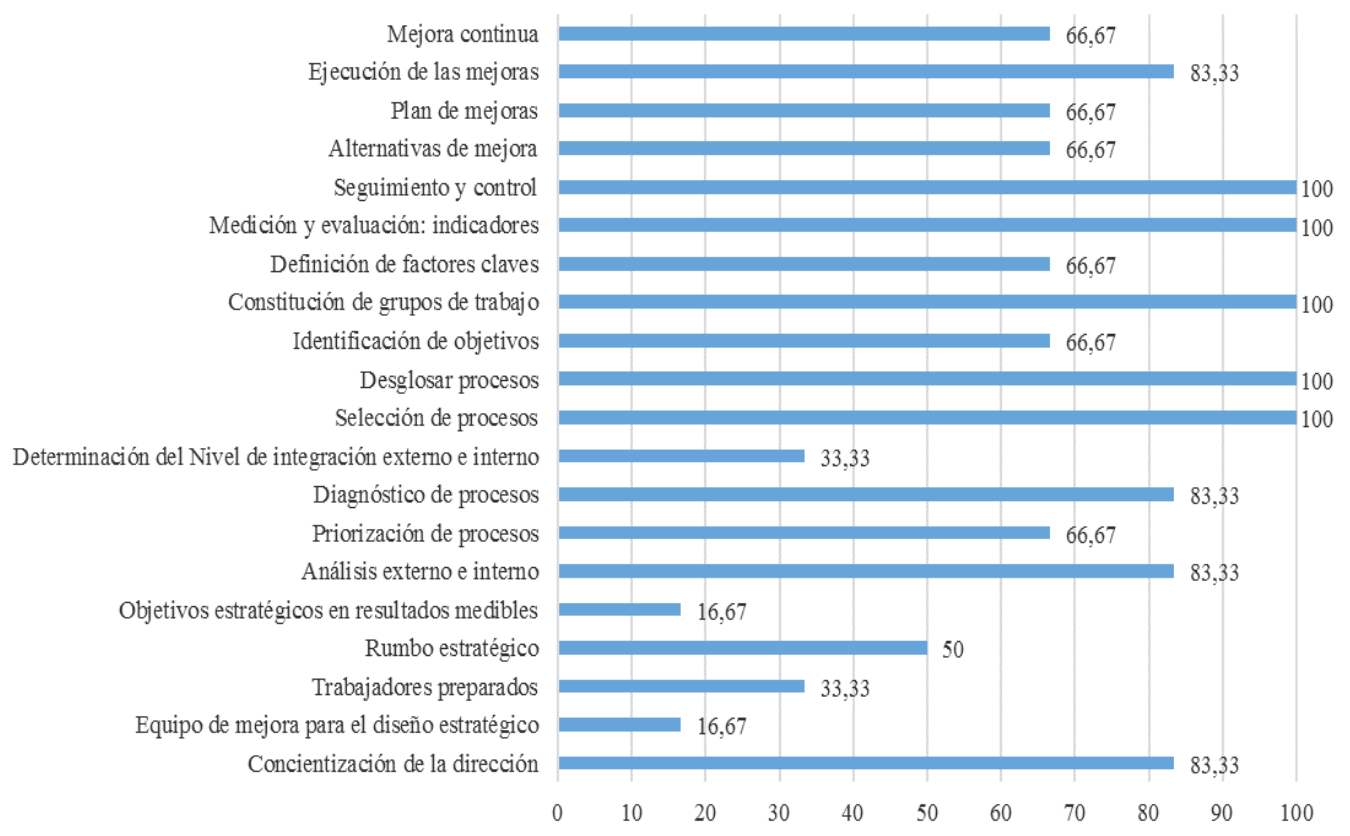

Figura 1. Nivel de inclusión de cada elemento en las metodologías analizadas 


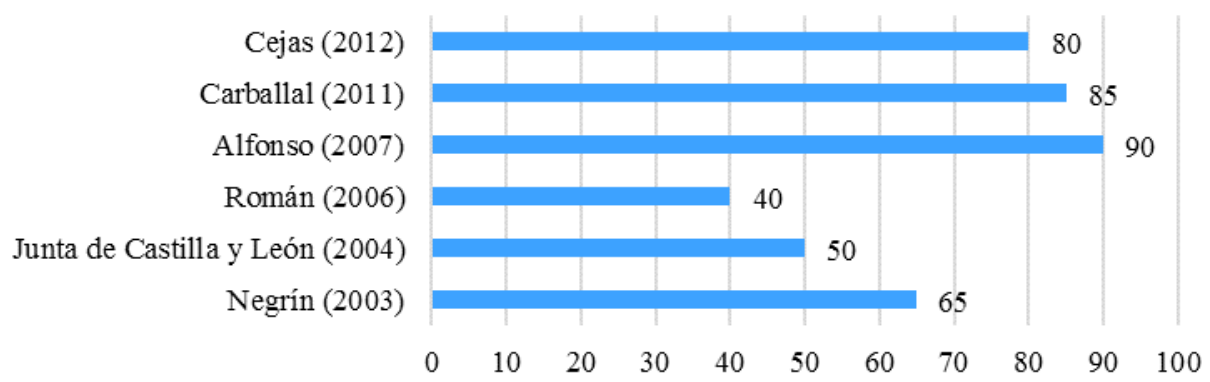

Figura 2. Porciento de elementos que incluye cada metodología

Para la inclusión en la metodología del enfoque de riesgos se revisaron los requisitos de gestión de la norma ISO/IEC 31010:2015, las técnicas de apreciación del riesgo del modelo DE-ARISDE (Bolaño et al., 2014), el método de identificación-medición-evaluación de riesgos para la Dirección Estratégica (Bolaño et al., 2011), y los aportes de José Carlos Melo en su libro Gestión de riesgos en la Organización: teoría y práctica (Melo, 2015).

La metodología propuesta incluye siete premisas, que definen supuestos a considerar ante circunstancias o condiciones futuras que podrán afectar la implementación de la metodología diseñada (Murillo, 2012); en su elaboración se tuvo en cuenta el Modelo DEISDE (Alfonso, 2007), así como del análisis de las metodologías estudiadas.

\section{Premisas:}

Sistema de Gestión de la Calidad implementado en la Organización

Contexto organizacional que refuerce las habilidades para el trabajo en equipo

Reconocimiento de la necesidad de una Gestión por Procesos

Compromiso de la Alta Dirección y miembros del Organización para proyectar, implantar y controlar la estrategia de cambio y dar seguimiento a la Gestión por Procesos

Proceso de dirección de la Organización basado en un Modelo de Dirección Estratégica

Desarrollo de consenso sobre la necesidad del cambio en la Organización hacia la integración de los sistemas de gestión

Trabajadores preparados

\section{Diseño metodológico:}

Se consideró que todos los elementos expuestos en cada una de las metodologías se podían agrupar en cuatro fases o etapas que abarcaran todas las tareas propuestas. En la Figura 3 se muestra el diseño metodológico propuesto. 


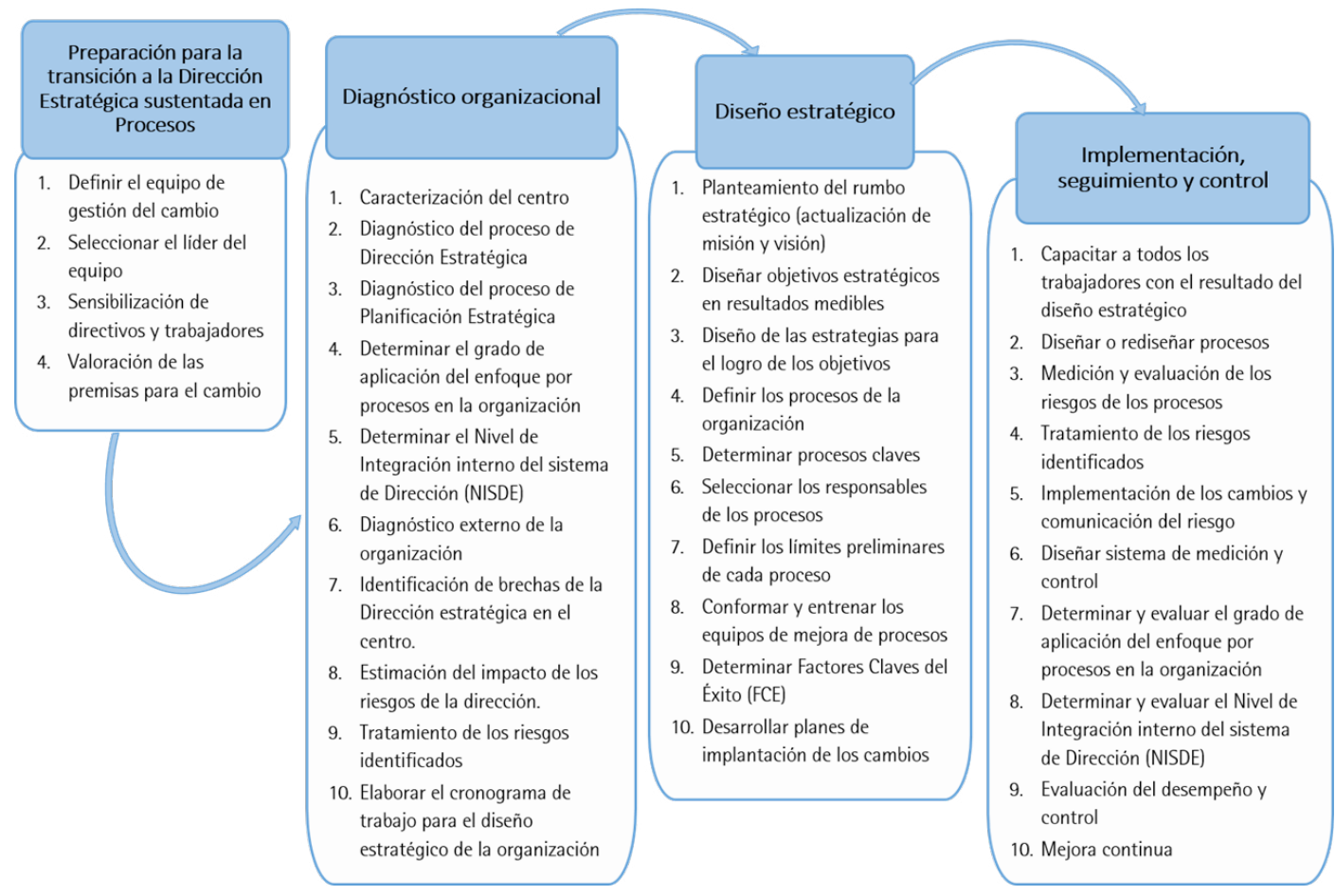

Figura 3. Metodología para la transición a un modelo de Dirección Estratégica sustentada en procesos

\section{Descripción de la metodología:}

Fase I: Planificación para la transición a un modelo de Dirección Estratégica sustentada en Procesos

Objetivo: Seleccionar y capacitar al equipo que va a realizar el cambio de la Organización hacia la Dirección Estratégica sustentada en Procesos, y valorar las premisas para la transición.

La importancia de esta etapa radicó en que es imprescindible preparar y sensibilizar a las personas que lideran todo el proceso de cambio en la Organización, y concientizar la necesidad del cambio en la mente de los directivos; con vistas a lograr que el proceso de transición al nuevo modelo de gestión que se propone se lleve a cabo de forma eficiente.

\section{Paso 1: Definición el equipo de gestión del cambio}

En este paso se tuvieron en cuenta determinados requisitos para seleccionar a las personas posibles de conformar el equipo de expertos: nivel de calificación en lo referente a la Dirección Estratégica y Gestión por Procesos, para ofrecer valoraciones conclusivas acerca de la problemática planteada con un máximo de competencia; años de experiencia; conocimiento del trabajo que desempeñan; cargo que ocupan en la organización; y preparación en la gestión de riesgos en el contexto estratégico.

\section{Paso 2: Selección del líder del equipo}

Para la selección del líder del equipo de cambio se tuvieron en cuenta los resultados obtenidos a partir del análisis del coeficiente de competencia, se estableció como requisito: que formara parte de la Alta Dirección preferiblemente con más de 10 años de experiencia en la 
Organización, que poseyera habilidades directivas, amplios conocimientos referentes a la Dirección por Procesos y a la Gestión de riesgos, así como buenas habilidades comunicativas, ya que es el encargado de garantizar la comunicación a todos los niveles.

\section{Paso 3: Sensibilización de los directivos y trabajadores}

En este paso se llevaron a cabo acciones para sensibilizar a las personas para que reconocieran la necesidad de cambio y desarrollaran consenso sobre la misma, motivados por un reto de superación y perfeccionamiento constantes. Se desarrollaron talleres, seminarios, y otras iniciativas, con el objetivo de movilizar y organizar a los trabajadores, por lo que primó el trabajo en grupo. Este paso se apoyó en especialistas externos que pudieran brindar su experiencia en relación con el proceso de transición.

\section{Paso 4: Valoración de las premisas para el cambio}

Se empleó el Método Delphi para la valoración de las premisas propuestas por parte de los expertos seleccionados. Posteriormente se demostró la confiabilidad en la consulta a los expertos mediante un análisis de concordancia utilizando el coeficiente de concordancia de Kendall (W). Se empleó el paquete estadístico MINITAB versión 15.1 para demostrar la concordancia entre los especialistas a partir de este coeficiente.

\section{Fase II: Diagnóstico organizacional}

Objetivo: Diagnosticar interna y externamente la organización para tener una visión global de los problemas que afectan la integración del Sistema de Dirección y las brechas que existen con las organizaciones tomadas como referencia para el estudio.

Durante esta etapa el trabajo principal que realizó el equipo de expertos consistió en la identificación y evaluación de una serie de categorías básicas asociadas a la Dirección por Procesos relacionadas con la propia Organización.

\section{Paso 1: Caracterización de la Organización}

En este paso se emplearon fundamentalmente la observación, la entrevista no estructurada y la revisión de documentos referentes a la Organización; se analizó el organigrama y el mapa de procesos, y se utilizaron representaciones gráficas para facilitar el análisis de los elementos de interés para la investigación.

\section{Paso 2: Diagnóstico del proceso de Dirección Estratégica}

El diagnóstico de este proceso se realizó mediante la aplicación a los expertos de la lista de chequeo propuesta por Alfonso (2007), para identificar las brechas entre el proceso de Dirección Estratégica de la Organización, tomando como patrón de referencia el modelo DEISDE. En este paso se empleó el coeficiente de concordancia de Kendall, que constituye una medida de coincidencia entre las valoraciones brindadas por los expertos con relación a la lista de chequeo.

\section{Paso 3: Diagnóstico del proceso de Planificación Estratégica}

Para diagnosticar el proceso de Planificación Estratégica se analizó el comportamiento del mismo en un período de tiempo que oscila entre tres y cinco años, el análisis abarcó los indicadores de desempeño en el período determinado, el flujograma y la ficha del proceso, tomando como base los requisitos establecidos por el organismo rector para la consecución 
del proceso.

\section{Paso 4: Determinación del grado de aplicación del enfoque por procesos}

Se empleó la lista de chequeo propuesta por Delgado (2011), la cual está conformada por doce preguntas donde la evaluación refleja el nivel de enfoque a procesos (NEP) existente en la Organización, y se aplicó a los especialistas de los procesos. A partir de las puntuaciones obtenidas según la valoración de los expertos se tabularon los datos y se determinó la concordancia entre los especialistas mediante el coeficiente de Kendall. Se asumieron las siguientes alternativas: si el NEP es mayor al 90\%, el grado de aplicación se considera Adecuado; si oscila entre el $50 \%$ y el $89 \%$, se considera Medio; y si es inferior al $49 \%$ se considera Muy Bajo. En general cuando el grado de aplicación es muy bajo, corresponde a una Organización que trabaja de manera funcional.

\section{Paso 5: Determinación del Nivel de Integración interno del sistema de Dirección (NISDE)}

Para calcular el Nivel de Integración entre los procesos de la Organización se establecieron una serie de consideraciones que, de forma general, consistieron en: definir todos los procesos que se realizan (teniendo en cuenta el mapa de procesos actual); confeccionar una matriz de interrelación entre los procesos; realizar entrevistas a los especialistas de procesos para establecer los flujos logísticos entre los procesos, y conocer la importancia y desempeño de ese proceso para el resto en una escala de cinco niveles (1-Nada importante, 2-Poco importante, 3-Medianamente importante, 4-Importante; 5-Muy importante); buscar las relaciones críticas ( $\geq 3$ y $D \leq 3$ ); y calcular el NISDE interno entre procesos propuesto por Alfonso (2007).

\section{Paso 6: Diagnóstico externo de la organización}

En este paso se empleó la técnica del Benchmarking, que no es más que fijar patrones, hitos o modelos a estudiar para realizar comparaciones que permitan evaluar la excelencia de la Organización. Para determinar los modelos de referencia se identificaron quiénes eran los grupos líderes en el sector de la ciencia, así como su proyección estratégica, para lo cual se requirió conocer cómo se organizaban, cómo era el diseño estratégico; y otros elementos de interés para la Organización.

\section{Paso 7: Identificación de brechas de la Dirección Estratégica}

Teniendo en cuenta los resultados de la lista de chequeo y de las brechas identificadas como parte del diagnóstico externo de la Organización se determinaron los elementos que limitaban el proceso de Dirección Estratégica, los cuales constituyen riesgos para el establecimiento del rumbo estratégico. Para analizar las interrelaciones de los elementos limitantes se emplearon los diagramas y matrices de relaciones propuestas por Alfonso (2007) que promueven el análisis de las relaciones causa-efecto de los elementos del proceso de Dirección Estratégica, con el objetivo de facilitar el análisis integrado de la situación actual del Organización.

\section{Paso 8: Estimación del impacto de los riesgos de la dirección}

Los riesgos identificados se relacionaron con los resultados del desempeño anterior de la Organización, las debilidades y amenazas identificadas en el diagnóstico estratégico, las relaciones entre los procesos y de otros aspectos que considere la Organización. Se realizó la estimación del impacto de los riesgos mediante el criterio de expertos a partir de la aplica- 
ción de métodos basados en evidencia considerando seis niveles, caracterizando cada uno de forma cualitativa según la propuesta de Melo (2015), tal y como se muestra en la Tabla 1.

Tabla 1. Estimación cualitativa del impacto de los riesgos.

\begin{tabular}{|c|l|}
\hline Valor cualitativo & \multicolumn{1}{c|}{ Descripción del impacto } \\
\hline 0 & No hay impacto \\
\hline Muy bajo & Muy bajo impacto en la gestión de la Organización \\
\hline Bajo & $\begin{array}{l}\text { Incidencias en la gestión de la Organización que implica pérdidas eco- } \\
\text { nómicas bajas. }\end{array}$ \\
\hline Medio & $\begin{array}{l}\text { Afectación en la gestión interna de la Organización que implica pérdi- } \\
\text { das económicas medianas. }\end{array}$ \\
\hline Alto & $\begin{array}{l}\text { Afectación en la gestión interna de la Organización que implica pérdi- } \\
\text { das económicas grandes. }\end{array}$ \\
\hline Muy alto & $\begin{array}{l}\text { Afectación relevante en la gestión de la Organización que implica pér- } \\
\text { didas económicas muy grandes, de reputación o de imagen. }\end{array}$ \\
\hline
\end{tabular}

Fuente: Melo (2015)

\section{Paso 9: Tratamiento de los riesgos identificados}

La NC ISO/IEC 31010:2015 establece que la decisión sobre el tratamiento del riesgo puede depender de los costos y de los beneficios de aceptar el riesgo, y de implantar controles mejorados. Según Melo (2015) el tratamiento de riesgo se define como el proceso de diseño, selección e implantación de medidas para modificar el nivel o valor de riesgo estimado. Aunque no se considera dentro de los aspectos relacionados con este, la aceptación del riesgo se podría tomar como la primera manifestación del tratamiento, pues con esta decisión la Organización acepta o asume un Nivel de Riesgo determinado en el marco de su desempeño:

Evitación del riesgo: Requiere que se haga un análisis para tomar las medidas necesarias a partir de cambios en métodos de trabajo o estructura de la Organización que eliminen la posibilidad de ser parte de situaciones evaluadas como peligrosas dentro del marco de su gestión.

Optimización del riesgo: Se lleva a cabo para disminuir la frecuencia de ocurrencia, el impacto del riesgo, y aumentar la detección a través de medidas preventivas en los procesos, las cuales deben tener un análisis de costo-beneficio sobre la base del cumplimiento de requisitos legales y normativos.

Traspaso del riesgo: Es el proceso de repartir con otras organizaciones la carga de pérdidas de un riesgo determinado. El traspaso del riesgo se ejecuta de forma general, a través de un contrato de seguro que recoge las principales obligaciones de las partes.

Retención del riesgo: Es una variante de la aceptación del riesgo, por tanto, se debe llevar a cabo un análisis de qué se acepta en cuanto a Nivel de Riesgo, a partir de las condiciones propias de la Organización, su objeto empresarial o encargo estatal.

\section{Paso 10: Elaboración del cronograma de trabajo para el diseño estratégico de la organización}

En este paso se registró cronológicamente la programación de las actividades correspondientes a la etapa de diseño estratégico. Se utilizó el Diagrama de Gantt para realizar la pro- 
gramación de cada uno de los pasos a realizar como parte de la Fase III referente al diseño estratégico. Se empleó el Microsoft Office Project 2016, el cual facilita la conformación del programa general y su monitoreo, ya que permite conocer los responsables en cada caso, así como las fechas de inicio y terminación de cada actividad.

Fase III: Diseño estratégico

Objetivo: Determinar la nueva proyección estratégica de la Organización

Para llevar a cabo de forma eficiente esta etapa, se tomaron como base en cuenta todas las brechas y debilidades identificadas en la fase anterior, tanto desde el punto de vista interno y externo, ya que aportaron los elementos que se deberán considerar en el nuevo diseño estratégico en busca de la excelencia e integración en la gestión. Durante esta fase se tuvieron en cuenta los riesgos relacionados con la estrategia de la Organización que se materializan en los procesos, afectando a los objetivos estratégicos formulados e impactando en el cumplimiento de la misión y el no alcance de la visión.

\section{Paso 1: Planteamiento del rumbo estratégico (actualización de Misión y Visión)}

Este paso es de suma importancia ya que la Misión es la razón de ser de la Organización y su formulación tiene consecuencias en la estructura. Por otro lado, la Visión considera aquello que se desea alcanzar a largo plazo, es la capacidad de ver más allá. La visión bien definida, dirigida y comunicada, motiva al personal a cumplir la razón de ser de la organización, es decir, su misión.

En este paso primó el trabajo en grupo, se realizaron sesiones de tormenta de ideas dividiendo a los participantes en grupos. Las ideas propuestas por cada grupo se comunicaron al resto de los equipos conformados con el objetivo de buscar los puntos coincidentes y por consenso formular una nueva misión y visión de la Organización, colectivamente aceptada.

\section{Paso 2: Diseño objetivos estratégicos en resultados medibles}

A criterio de varios autores en la bibliografía, los objetivos eficaces tienen las siguientes características: especificidad, asequibilidad, mensurabilidad, orientados a resultados y limitados en el tiempo. Los objetivos trasladan la misión a términos concretos para cada nivel de la organización, por lo que deben guardar coherencia con la misión y con el análisis interno y externo. Deben estar enfocados a obtener resultados específicos en términos económicos y financieros, de satisfacción de necesidades, de procesos y de desarrollo humano.

\section{Paso 3: Diseño de estrategias para el logro de los objetivos}

Las estrategias son los planteamientos que sirven para cumplir la misión de la Organización, o sea, se determinaron los objetivos a largo plazo y se eligieron las acciones y se asignaron los recursos necesarios para conseguirlos. La Alta Dirección fue la encargada de definir las estrategias ya que constituyen una guía de direccionamiento para alcanzar los objetivos, y de este modo convertirse en la Organización que se desea ser en un futuro.

\section{Paso 4: Definición de los procesos de la Organización}

El equipo de expertos, apoyándose en otros especialistas y a través de la técnica de tormentas de ideas valoró cuáles procesos pueden ser eliminados o integrados con otros procesos, de forma tal que se logre el cumplimiento del rumbo estratégico de la Organización. 
Posteriormente se buscaron los macroprocesos (áreas de resultados claves) que contribuían esencialmente al logro de los objetivos estratégicos y a la misión de la organización, y los procesos relevantes (de tres a diez procesos) que se incluyeron en cada macroproceso. Para el establecimiento de los macroprocesos se realizaron sesiones de trabajo en grupo con directivos de la Organización, así como representantes de las diferentes áreas funcionales de la Organización y responsables de procesos.

\section{Paso 5: Determinación de procesos clave}

Los procesos clave se consideren esenciales para el rendimiento efectivo en la Organización; los logros dentro de estos procesos son necesarios para llevar a cabo con éxito su Misión y para que cumpla con las expectativas generadas. Si bien estos procesos no llegan a cubrir todo lo que logrará la Organización, identifican los aspectos significativos de los cuales depende su desempeño. Se establecieron los criterios que consideró la Organización (impacto, cliente, resultados, entre otros), de forma tal que se pudo conformar una matriz de prioridades para determinar el orden de prioridad con que fueron estudiados y analizados los procesos clave.

\section{Paso 6: Selección de los responsables de los procesos}

El responsable del proceso se convierte en un directivo integrador con poder de decisión sobre el proceso, pero fue necesario que el equipo de cambio y los directivos reconocieran que no necesariamente tenía que ser uno de los directivos funcionales que ejercen su poder jerárquico en la Organización. Se consideraron aquellos miembros que, aunque eran o no dirigentes, se sentían identificados con el proceso y tenían una buena comunicación, ya que necesita relacionarse con las diferentes áreas o unidades organizativas que intervienen en el proceso, manteniendo el control en su conjunto.

\section{Paso 7: Definición de los límites preliminares de cada proceso}

Cada responsable estableció los límites preliminares de su proceso, o sea, dónde comenzaba y terminaba, lo cual estuvo en correspondencia con los criterios de las diferentes áreas y personas involucradas. Durante este paso, se llevaron a cabo sesiones de trabajo en grupo, con el objetivo de lograr un consenso en relación a los límites preliminares, que fueron establecidos oficialmente como resultado final de este paso.

\section{Paso 8: Conformación y entrenamiento de los equipos de mejora de procesos (EMP)}

Los equipos de proceso tuvieron entre seis y doce miembros que representaban todas las disciplinas funcionales interesadas, y que además estaban bien relacionados con la misión, objetivos y estrategia de la Organización. Se impartieron talleres, seminarios y cursos para capacitar en habilidades básicas de trabajo en equipo, aplicación de técnicas participativas, de solución de problemas y toma de decisiones en grupo.

\section{Paso 9: Determinación de Factores Claves del Éxito (FCE)}

Los FCE constituyen un elemento de medición para evaluar la eficacia de un proyecto de mejora y se definen por los requerimientos que le imponen al proceso todas aquellas personas, grupos u Organizaciones a las cuales afecta, tanto de forma positiva como negativa. Estos factores se determinaron a partir de ideas sobre la base de la experiencia de los implicados, por lo que se desarrollaron sesiones de tormenta de ideas. El número de factores clave detectados no fue excesivo, ya que puede complicar el análisis posterior, de ahí la conveniencia 
de reducir el listado de los factores clave a no más de cinco o seis.

\section{Paso 10: Desarrollo de planes de implantación de los cambios}

Los planes partieron de definir una misión del EMP, que definía exactamente la razón de ser del equipo. El cronograma de ejecución detalló las diferentes actividades que el EMP debía desarrollar y los resultados que se debían alcanzar en cada una, para lo cual se empleó el Diagrama de Gantt.

Fase IV: Implementación, seguimiento y control

Objetivo: Ejecutar los cambios previstos en los procesos y diseñar los nuevos sistemas de medición y control.

Esta fase se ejecutó por parte de un Equipo de Implementación del Proceso (EIP), donde participaron todos los miembros del EMP, pero también estuvieron constituidos por nuevos miembros, sobre todo por aquellos que tenían una incidencia directa en la ejecución del proceso diseñado o rediseñado.

Como parte de esta fase, se implementaron todos los cambios realizados en la fase anterior de la metodología referente al Diseño Estratégico. Se diseñó el sistema de medición y control, se determinó la eficacia de los cambios realizados, y se garantizó la retroalimentación inmediata de los resultados mediante el último paso propuesto que lo constituye la mejora continua.

\section{Paso 1: Capacitación a todos los trabajadores con el resultado del diseño estratégico}

Es necesario que todos los trabajadores comprendan y se sientan identificados con la nueva misión y visión, teniendo siempre como premisa que el factor humano es decisivo para la consecución de las mismas. Se realizaron talleres, seminarios y sesiones de trabajo con todos los trabajadores, y se aplicaron de encuestas y entrevistas para determinar el nivel de compromiso de los mismos.

\section{Paso 2: Diseño o rediseño de procesos}

Como parte de este paso se elaboró el diagrama de flujo del proceso, se identificaron las actividades de valor agregado para evaluar el impacto de cada actividad del proceso sobre los FCE, se calcularon las oportunidades de mejora, y se aplicó benchmarking al proceso con vistas a elaborar un nuevo diseño del mismo, tomando en cuenta los objetivos estratégicos establecidos y también los FCE.

En este punto se llevó a cabo un análisis de mejoramiento en cuanto a costos y riesgos, previo a la implementación del proceso. Entre las herramientas que se usaron en la identificación de riesgos se encuentran Diagrama Ishikawa, IDEFO, Listas de chequeo de los procesos, Árboles de fallas, Árboles de eventos, Análisis de puntos críticos de control, entre otros.

\section{Paso 3: Medición y evaluación de los riesgos de los procesos}

En este paso se tuvieron en cuenta la propuesta de la ISO 31000:2009, el Método de identificación-medición y evaluación de riesgos de Bolaño et al. (2011), y el Modelo de administración de riesgos DE - ARISDE de Bolaño et al. (2014), ya que estas metodologías son las que incluyen la mayor cantidad de elementos a considerar como parte de la gestión de riesgos. Sobre esta base se elaboró un procedimiento para la administración de riesgos en el centro 
para llevar a cabo la medición y evaluación de estos tanto cualitativa como cuantitativamente.

\section{Paso 4: Tratamiento de los riesgos identificados}

En este paso se tuvieron en cuenta las consideraciones establecidas por Melo (2015), las cuales se esclarecieron en el Paso 9 de la Fase II.

\section{Paso 5: Implementación de los cambios y comunicación del riesgo}

El EIP preparó un plan detallado y fue el encargado de coordinar los cambios que se realizaron, quedando documentados en los procedimientos de trabajo. Este equipo mantuvo un estrecho control sobre cada cambio, con el fin de asegurar que se implementara de forma correcta.

La comunicación del riesgo como parte integrante del sistema de comunicación de la Organización implicó una constante relación entre los trabajadores y los diferentes niveles de dirección, con el objetivo de conocer los cambios provocados en el análisis de los procesos, el desempeño de estos, los riesgos identificados, su estimación y medidas de tratamiento, logrando una mejora continua en la gestión de los riesgos determinados.

\section{Paso 6: Diseño del sistema de medición y control}

A criterio de Carballal (2011) antes de que la Organización pueda diseñar un sistema de medición es necesario definir requerimientos e indicadores concretos que permitan evaluar el desempeño. Como parte de este paso se propuso la implementación del Cuadro de Mando Integral (CMI) como herramienta de gestión para el establecimiento de los indicadores que conciernen a la Organización. Es costumbre en las organizaciones tener un número determinado de indicadores para los objetivos, y por otro lado otro grupo de indicadores para medir y evaluar la eficacia de los procesos determinados para la gestión. Se hizo un análisis de estos, para dar un sentido de integración a los mismos, que contribuyó a la disminución de tiempo en análisis, viendo los problemas y soluciones de manera integral .

\section{Paso 7: Determinación del grado de aplicación del enfoque por procesos}

Se empleó la lista de chequeo propuesta por Delgado (2011) teniendo en cuenta las consideraciones establecidas por el propio autor, las cuales quedaron plasmadas en el Paso 4 de la Fase II.

\section{Paso 8: Determinación del Nivel de Integración interno del sistema de Dirección (NISDE)}

Para el cumplimiento de este paso se calculó el indicador propuesto por Alfonso (2007) basado en las relaciones de los procesos. Se tuvieron en cuenta las consideraciones establecidas como parte del Paso 5 de la Fase II de esta metodología, pero en este caso se tomaron como referencia los nuevos procesos establecidos.

\section{Paso 9: Evaluación del desempeño y control}

Lo que se perseguía en este paso es el análisis crítico de los resultados ofrecidos a partir de la aplicación de la lista de chequeo para determinar el grado de aplicación del enfoque por procesos y del cálculo del NISDE, con el objetivo de conocer si los cambios realizados contribuyeron a incrementar el nivel de enfoque por procesos, así como a la integración de los procesos de la organización. 


\section{Paso 10: Mejora continua}

En este paso se consideró que el cambio realizado había llegado a concretar una significativa ruptura en el desempeño de la organización, y a partir de aquí, esta no puede dejar de mejorar. Es necesario obtener información directa por parte de los clientes, tanto internos como externos, ya que son los únicos capaces de evaluar si las actividades del proceso se ejecutan de acuerdo con sus requerimientos. La mejora continua se lleva a cabo por los propios equipos que trabajan en el proceso, que serán los encargados de aportar las ideas para su mejora, por ser los que están directamente relacionados con sus clientes.

\section{RESULTADOS}

La metodología se aplicó en una entidad de Ciencia, Tecnología e Innovación para conducir la elaboración y establecimiento del Plan Estratégico para el período 2017-2021, a continuación, se relacionan de forma general los principales resultados obtenidos en cada una de las fases.

\section{Fase I: Planificación para la transición a un modelo de Dirección Estratégica susten-} tada en Procesos

Se aplicó una encuesta a 9 expertos para determinar el grado de cumplimiento de las premisas propuestas. El procesamiento de los datos se realizó a partir del empleo del Método Delphi, mediante el programa estadístico Método de Consultas a Expertos. De acuerdo a los resultados obtenidos todas las premisas propuestas son consideradas como Muy Adecuadas, existiendo una concordancia entre los expertos del $66.5 \%$. Estos resultados corroboran que en el centro existen las condiciones necesarias para acometer el proceso de transición al modelo de Dirección Estratégica sustentada en Procesos.

\section{Fase II: Diagnóstico organizacional}

Al aplicar las herramientas propuestas en cada uno de los pasos se determinó que el nivel de enfoque a procesos es de $60.19 \%$, por lo que el grado de aplicación se considera medio; y existe un bajo Nivel de Integración interna del Sistema de Dirección (0.23).

Las principales brechas identificadas tomando como referencia el Modelo DEISDE, están relacionadas con los equipos de mejora, diagnóstico, diseño y rediseño de procesos clave y funcionales, y la supervisión y ajuste.

Para la cuantificación de los riesgos asociados a las amenazas y debilidades identificadas se aplicó un procedimiento para la administración de riesgos en la Organización, tomando como base de la propuesta de la ISO 31000:2009, el Método de identificación-medición y evaluación de riesgos de Bolaño et al. (2011), y el Modelo de administración de riesgos DE - ARISDE de Bolaño et al. (2014). El procedimiento realizó un análisis cualitativo y cuantitativo de los valores de riesgos estimados, tanto desde el punto de vista estratégico como del económico-financiero, y es aplicable a todos los procesos y actividades de un Sistema de Gestión.

Fase III: Diseño estratégico

A partir de los resultados obtenidos en el diagnóstico se definió el nuevo diseño estratégico 
de la organización, a continuación, se relacionan los aspectos fundamentales relacionados con esta fase:

Misión: Somos un centro de Ciencia, Tecnología e Innovación que contribuye al desarrollo sostenible de la sanidad animal, vegetal y humana, a partir de la ejecución de proyectos básicos y de innovación tecnológica, formación académica, desarrollo de productos y servicios científico técnicos especializados, con un personal altamente calificado y comprometido.

Visión: Ser un centro de referencia nacional y en la región de Latinoamérica y el Caribe para la generación de conocimientos, formación académica, desarrollo de productos y servicios científicos-técnicos competitivos para el sector agroalimentario, con enfoque a una Salud.

Procesos: Los procesos clave se hicieron corresponder en su nomenclatura con las Áreas de Resultados Claves del centro. La Figura 4 muestra el mapa de procesos diseñado.

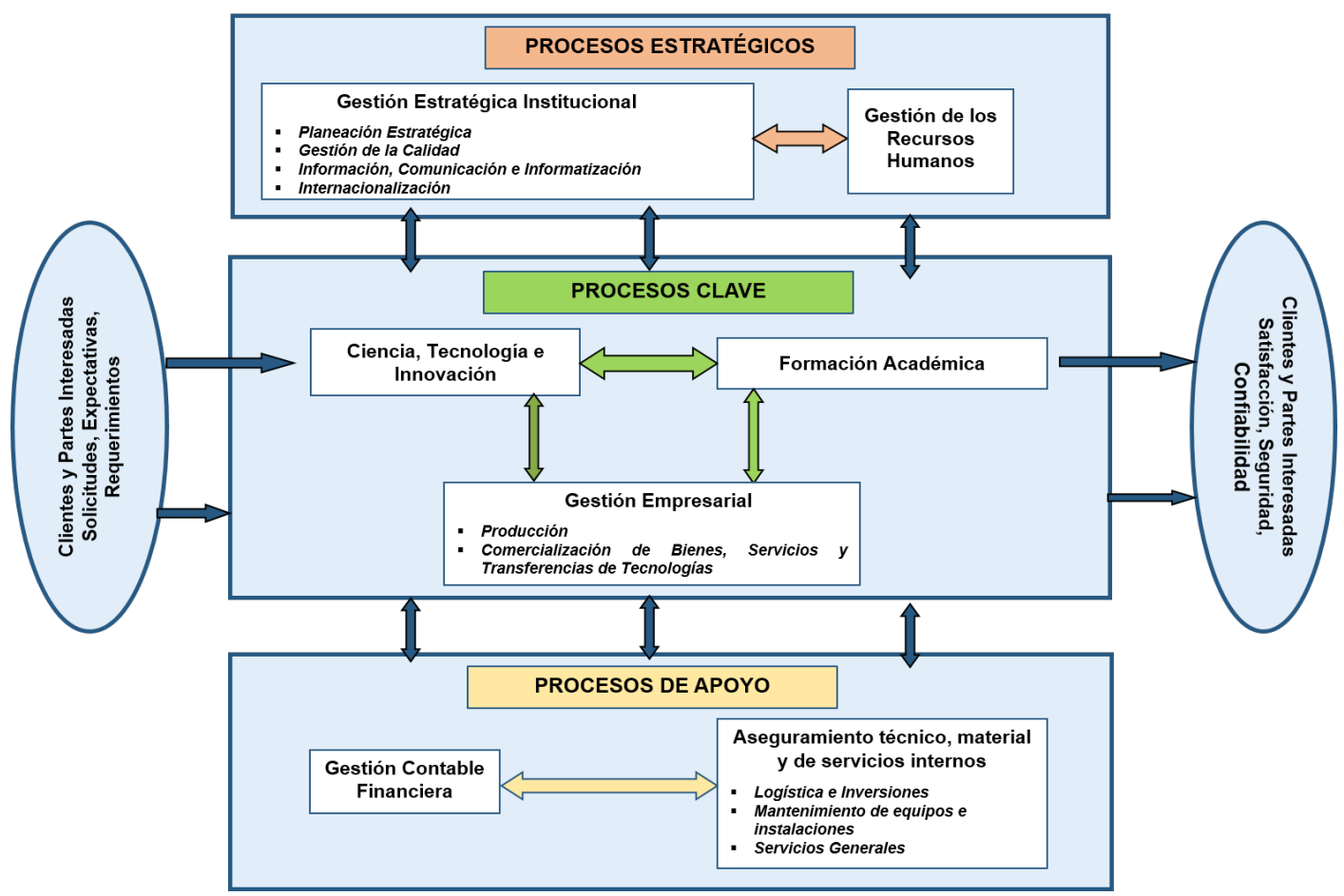

Figura 4. Mapa de procesos de la organización objeto de estudio

\section{Factores Claves de Éxito:}

- Incremento de los ingresos

- Reducción de los gastos

- Fidelidad y retención de Clientes

- Crecimiento del mercado

- Rapidez y Flexibilidad ante los cambios del entorno

- Tecnologías, productos y servicios científico-técnicos con alto valor agregado

- Fuerte Sistema de Información y Comunicación para la Toma de Decisiones.

- Personal competente y motivado 
- Experiencia y reconocimiento en la investigación científica y Formación académica

\section{Fase IV: Implementación, seguimiento y control}

Actualmente en la entidad objeto de estudio está en fase de elaboración de un procedimiento para el diseño de un sistema de Control de Gestión apoyado en el Cuadro de Mando Integral (CMI) como herramienta fundamental para su diseño, tomando como referencia el mapa estratégico de indicadores globales identificados, el cual se muestra en la Figura 5, y está en correspondencia con los FCE previamente establecidos.

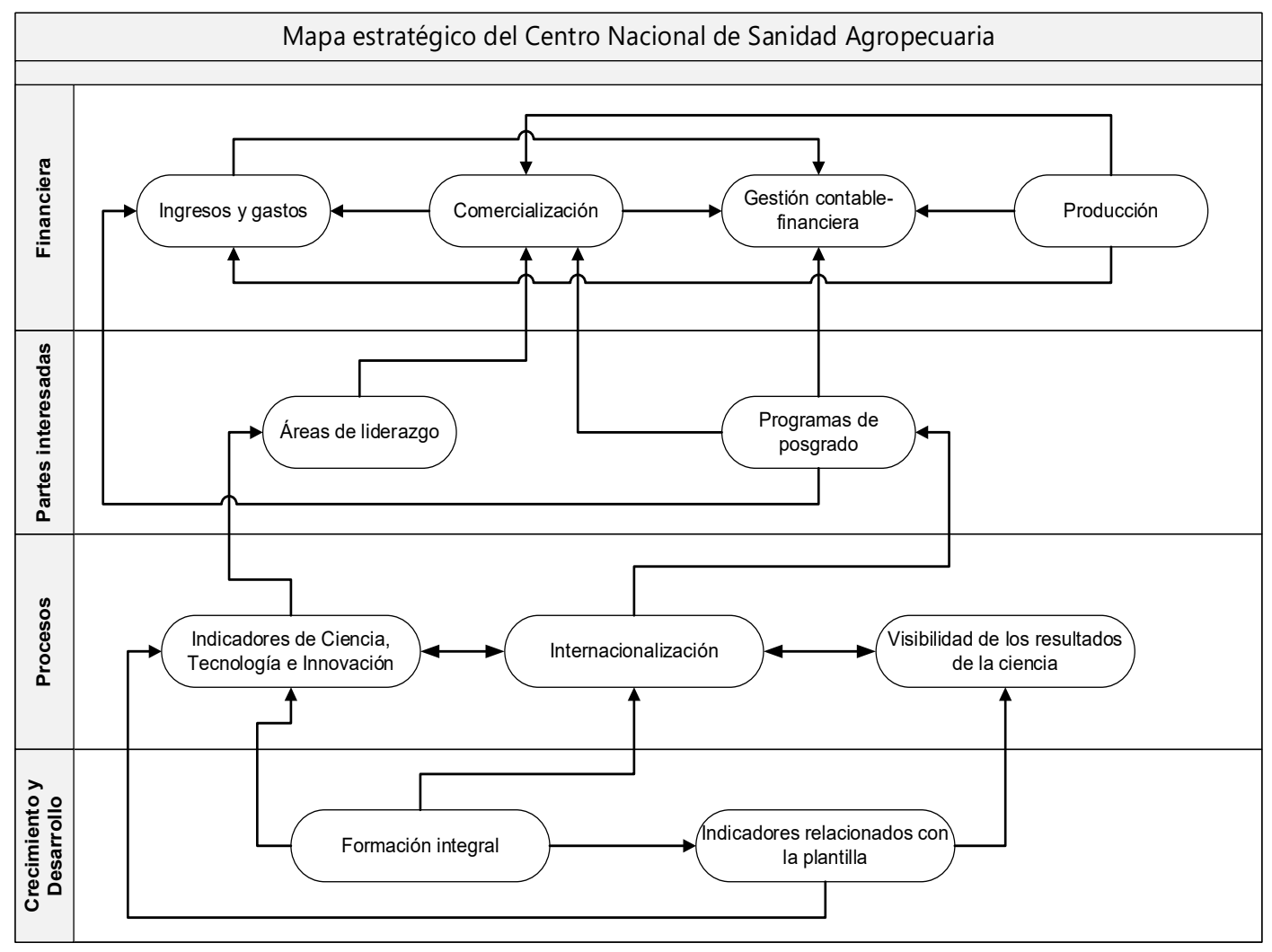

Figura 5. Mapa estratégico de la organización objeto de estudio

\section{DISCUSIÓN}

Para analizar las tendencias actuales de la Dirección Estratégica, se estudiaron 21 fuentes bibliográficas relacionadas con el campo de estudio de la Dirección Estratégica (Rummler \& Brache, 1995; Chiavenato, 2002; Kaplan \& Norton, 2005; Gary, 2005; Ramírez, 2005; MaIlette \& Hopkins, 2013; Fisher \& Oberholzer, 2013; Conley et al., 2013; Mitchell et al., 2013; Martin et al., 2013; Becerra et al., 2013; Moya et al., 2013; Valdés et al., 2013; Stachuk et al., 2013; Caballero et al., 2014; Choe et al., 2014; Bolaño et al., 2014; Vallejo et al., 2015; Álvarez \& Vizcaíno, 2015; Sánchez, 2016; Briones et al., 2017). En un análisis exploratorio de estos documentos se observó que las características de mayor atención por los investi- 
gadores se enfocan hacia: las mejoras en el proceso de Dirección Estratégica (PDE), énfasis en determinado tipo de estrategia $(E)$, enfoque de recursos, capacidades y competencias $(E R C)$, integración estratégica (IE), alineación estratégica (AE). Predominan las aplicaciones en empresas (ApE) por encima de las que se realizan en el sector público (ASP).

Por otro lado, solo 10 autores hacen referencia a la Gestión por Procesos (GP) (Rummler \& Brache, 1995; Kaplan \& Norton, 2005; Valdés et al., 2013; Becerra et al., 2013; Moya et al., 2013; Caballero, Gómez \& Díaz, 2014; Bolaño et al., 2014; Vallejo et al., 2015; Álvarez \& Vizcaíno, 2015; Briones et al., 2017) a pesar de la necesidad de lograr la integración en la cadena de procesos de la organización como un escaño superior del sistema de Dirección de las organizaciones, donde lo principal es satisfacer las necesidades crecientes de los clientes y la sociedad, y como consecuencia obtener niveles de eficacia y eficiencia superiores.

La Administración de riesgos (AR), es el aspecto menos tratado por los autores, siendo abordada solo por tres (Bolaño et al., 2014; Vallejo et al., 2015; Sánchez, 2016), lo cual contrasta con la necesidad de desarrollar la administración de riesgos, como una parte integral del proceso de administración, para lograr mayor anticipación frente a desafíos y oportunidades que se generan de las condiciones externas y de la realidad interna.

La Tabla 2 muestra la inclusión de los aspectos previamente mencionados en las fuentes bibliográficas analizadas

Tabla 2. Estudio de fuentes bibliográficas relacionadas con el campo de estudio de la Dirección Estratégica.

\begin{tabular}{|l|c|c|c|c|c|c|c|c|c|}
\hline \multicolumn{1}{|c|}{ Fuente } & PDE & E & ERC & IE & AE & ApE & ASP & GP & AR \\
\hline Rummler y Brache (1995) & $\mathrm{X}$ & & & $\mathrm{X}$ & & $\mathrm{X}$ & & $\mathrm{X}$ & \\
\hline Chiavenato (2002) & $\mathrm{X}$ & $\mathrm{X}$ & $\mathrm{X}$ & $\mathrm{X}$ & $\mathrm{X}$ & $\mathrm{X}$ & & & \\
\hline Kaplan y Norton (2005) & $\mathrm{X}$ & $\mathrm{X}$ & $\mathrm{X}$ & & $\mathrm{X}$ & $\mathrm{X}$ & & $\mathrm{X}$ & \\
\hline Gary (2005) & & $\mathrm{X}$ & $\mathrm{X}$ & & & $\mathrm{X}$ & & & \\
\hline Ramírez (2005) & & $\mathrm{X}$ & $\mathrm{X}$ & & & & & & \\
\hline Mallette y Hopkins (2013) & & $\mathrm{X}$ & $\mathrm{X}$ & & & & & & \\
\hline Fisher III y Oberholzer-Gee (2013) & & $\mathrm{X}$ & $\mathrm{X}$ & & & $\mathrm{X}$ & & & \\
\hline Conley et al. (2013) & & $\mathrm{X}$ & $\mathrm{X}$ & & $\mathrm{X}$ & & & & \\
\hline Mitchell et al. (2013) & & $\mathrm{X}$ & & & & $\mathrm{X}$ & & & \\
\hline Martin et al. (2013) & $\mathrm{X}$ & & & & $\mathrm{X}$ & $\mathrm{X}$ & & $\mathrm{X}$ & \\
\hline Valdés et al. (2013) & $\mathrm{X}$ & $\mathrm{X}$ & $\mathrm{X}$ & & & $\mathrm{X}$ & & & \\
\hline Stachuk et al. (2013) & $\mathrm{X}$ & $\mathrm{X}$ & & $\mathrm{X}$ & & & $\mathrm{X}$ & $\mathrm{X}$ & \\
\hline Becerra et al. (2013) & $\mathrm{X}$ & & & $\mathrm{X}$ & & & $\mathrm{X}$ & $\mathrm{X}$ & \\
\hline Moya et al. (2013) & $\mathrm{X}$ & $\mathrm{X}$ & & & & $\mathrm{X}$ & & $\mathrm{X}$ & \\
\hline Caballero et al. (2014) & & $\mathrm{X}$ & & & & & & & \\
\hline Choe et al. (2014) & $\mathrm{X}$ & & $\mathrm{X}$ & $\mathrm{X}$ & $\mathrm{X}$ & & & $\mathrm{X}$ & $\mathrm{X}$ \\
\hline Bolaño et al. (2014) & $\mathrm{X}$ & $\mathrm{X}$ & $\mathrm{X}$ & & & $\mathrm{X}$ & & $\mathrm{X}$ & $\mathrm{X}$ \\
\hline Vallejo et al. (2015) & $\mathrm{X}$ & $\mathrm{X}$ & $\mathrm{X}$ & $\mathrm{X}$ & $\mathrm{X}$ & & $\mathrm{X}$ & $\mathrm{X}$ & \\
\hline Álvarez y Vizcaíno (2015) & $\mathrm{X}$ & $\mathrm{X}$ & $\mathrm{X}$ & $\mathrm{X}$ & $\mathrm{X}$ & $\mathrm{X}$ & & & $\mathrm{X}$ \\
\hline Sánchez (2016) & $\mathrm{X}$ & $\mathrm{X}$ & $\mathrm{X}$ & $\mathrm{X}$ & $\mathrm{X}$ & & $\mathrm{X}$ & $\mathrm{X}$ & \\
\hline Briones et al. (2017) & $\mathbf{1 3}$ & $\mathbf{1 7}$ & $\mathbf{1 4}$ & $\mathbf{8}$ & $\mathbf{9}$ & $\mathbf{1 2}$ & $\mathbf{4}$ & $\mathbf{1 0}$ & $\mathbf{3}$ \\
\hline
\end{tabular}


Significado de los acrónimos: PDE: Mejoras en el proceso de Dirección Estratégica, E: Énfasis en determinado tipo de estrategia, ERC: Enfoque de recursos, capacidades y competencias, IE: Integración estratégica, AE: Alineación estratégica, ApE: Aplicaciones en empresas, ASP: Aplicaciones en el sector público, GP: Gestión por Procesos, AR: Administración de riesgos.

La metodología diseñada no solo incluye elementos de la Dirección Estratégica Integrada y la Gestión por procesos, sino que también incorpora el enfoque de riesgos, lo cual constituye un aspecto novedoso, ya que en la literatura consultada no existen precedentes de una metodología para llevar a cabo la transición de un modelo de Dirección por Objetivos a una Dirección sustentada en procesos, incluyendo la gestión de los riesgos en Organizaciones que basen su sistema de gestión en un modelo de Dirección Estratégica.

\section{CONCLUSIONES}

El problema formulado se consideró de gran actualidad y pertinencia, tanto en el plano conceptual-metodológico como práctico, debido a que integra coherentemente la Dirección Estratégica, la gestión por procesos y la administración de riesgos, pues en la literatura consultada resulta insuficiente el tratamiento metodológico dado de forma integrada a estos aspectos.

La metodología propuesta favorece la flexibilidad en las organizaciones, permitiendo mayor coherencia para realizar los cambios, mejorar el desempeño de la gestión, mayor anticipación frente a desafíos y oportunidades que se generan de las condiciones externas y de la realidad interna, para lograr mayor competitividad y sostenibilidad, sobre todo para instituciones localizadas en países en desarrollo.

La aplicación de la metodología en la organización objeto de estudio definió las principales brechas existentes y favoreció a la entidad de nuevo diseño estratégico, lo que que permite el incremento del desempeño y ofrece mayor flexibilidad para la anticipación al entorno.

\section{REFERENCIAS}

ALFONSO, D. Modelo De Dirección Estratégica Para La Integración Del Sistema De Dirección De La Empresa [en línea]. Tesis presentada en opción al grado científico de Doctor en Ciencias. S.I.: Instituto Superior Politécnico «Jose Antonio Echeverría», 2007. [Consulta: 3 abril 2016] Disponible en: http://catedragc.mes.edu.cu/download/Tesis\%20de\%20Doctorado/ Ingeniera\%20Industrial\%20-\%20Nacionales/DanielAlfonsoRobainaTESIS.pdf.

ALFONSO, D., VILLAZÓN, A., MILANES, P.E., RODRÍGUEZ, A. y ESPÍN, R. Procedimiento general de rediseño organizacional para mejorar el enfoque a procesos. Ingeniería Industrial [en línea]. 2011, 32(3), 238-248. [Consulta: 8 febrero 2017]. Disponible en: http://www. redalyc.org/pdf/3604/360433577010.pdf.

ÁLVAREZ, L.Q. y VIZCAÍNO, L.H. Diseño del proceso de Dirección Estratégica, cambio de un enfoque funcional a enfoque de proceso. Avances [en línea]. 2015, 15(3), 290-298. [Consulta: 27 diciembre 2017]. Disponible en: http://www.ciget.pinar.cu/ojs/index.php/publicaciones/ 
article/view/110.

BECERRA, A.A., FERNÁNDEZ, E.M. y ROBAINA, D.A. Dirección por procesos en la Universidad. Ingeniería Industrial, 2013, 34(1), 87-95.

BOLAÑO, Y., ALFONSO, D., PÉREZ, A. y ARIAS, M. Modelo de Dirección Estratégica basado en la Administración de Riesgos. Ingeniería Industrial [en línea]. 2014, 35(3), 344357. [Consulta: 8 mayo 2017]. Disponible en: http://scielo.sld.cu/pdf/rii/v35n3/rii10314.pdf.

BOLAÑO, Y., ALFONSO, D., RAMÍREZ, A. y HERNÁNDEZ, A.A. Método de Identificación medición y evaluación de riesgos para la Dirección Estratégica. Ingeniería Industrial [en línea]. 2011, 32(3), 162-169. [Consulta: 7 septiembre 2017]. Disponible en: http://www.redalyc.org/ pdf/3604/360433576011.pdf.

BRIONES, V.F.V., BECERRA, A.A., ROBAINA, D.A. y FERNÁNDEZ, E.S.M. Análisis estratégico de una universidad basado en el enfoque por procesos. Ingeniería Industrial [en línea]. 2017, 38(2), 201-209. [Consulta: 20 julio 2018]. Disponible en: http://scielo.sld.cu/ scielo.php?script=sci_abstract\&pid=S1815-59362017000200009\&lng=es\&nrm=iso\&t|ng=es.

CABALLERO, G.B., GÓMEZ, L.O. y DÍAZ, M.E.A. Diagnóstico para valorar la introducción de un nuevo servicio. Ingeniería Industrial, 2014, 35(1), 85-93.

CARBALLAL, E. Las estructuras colaborativas. El tránsito de las estructuras jerárquicas a las estructuras colaborativas. La Habana: Félix Varela. 2011. ISBN 978-959-07-1627-0.

CEJAS, C. Procedimiento para introducir la gestión por procesos. [en línea], 2012. Disponible en: http://www.gestiopolis.com/procedimiento-para-introducir-la-gestion-por-procesos/.

CHIAVENATO, I. Administración en los nuevos tiempos. Bogotá. Colombia: McGraw-Hill Interamericana. 2002. ISBN 978-958-41-0301-7.

CHOE, C., DEY, T. y MISHRA, V. Corporate diversification, executive compensation and firm value: Evidence from Australia. Australian Journal of Management [en línea]. 2014, 39(3), 395-414. [Consulta: 8 mayo 2017]. Disponible en: http://journals.sagepub.com/doi/ abs/10.1177/0312896213499027.

CIENFUEGOS, I. Risk Management, the integrated perspective, a model that can contribute to full fit the goals of modern Municipalities. Polytechnical Studies Review [en línea]. 2009, 7(11). [Consulta: 4 junio 2017]. Disponible en: http://www.scielo.mec.pt/pdf/tek/n11/n11a09. pdf.

CONLEY, J.G., BICAN, P.M. y ERNST, H. Value Articulation: A Framework for the Strategic Management of Intellectual Property. California Management Review [en línea]. 2013, 55(4), 102-120. [Consulta: 30 enero 2018]. Disponible en: http://journals.sagepub.com/doi/10.1525/ cmr.2013.55.4.102.

DELGADO, D. Enfoque a procesos, tendencias actuales. Madrid: Espasa-Calpe-CDN. 2011

FISHER, W.W. y OBERHOLZER, F.G. Strategic management of intellectual property: an integrated approach. California management review [en línea]. 2013, 55(4), 157-183. [Consulta: 19 febrero 2018]. Disponible en: http://journals.sagepub.com/doi/abs/10.1525/ cmr.2013.55.4.157.

GARY, M.S. Implementation strategy and performance outcomes in related diversification. Strategic Management Journal [en línea]. 2005, 26(7), 643-664. [Consulta: 27 abril 2018]. Disponible en: https://onlinelibrary.wiley.com/doi/epdf/10.1002/smj.468. 
HERNÁNDEZ, A., NOGUEIRA, D., MEDINA, A. y MARQUÉS, M. Inserción de la gestión por procesos en instituciones hospitalarias. Concepción metodológica y práctica. Revista de Administração [en línea]. 2013, 48(4), 739-756. [Consulta: 4 abril 2017]. Disponible en: http:// www.scielo.br/pdf/rausp/v48n4/09.pdf.

ISO 31000:2009. Gestión de Riesgos - Principios y Guías. 15 noviembre 2009.

ISO 9001:2015. Sistemas de gestión de la calidad - Requisitos. 15 septiembre 2015.

ISO/IEC 31010:2015. Gestión de riesgo - Técnicas de apreciación del riesgo. Marzo 2015

JUNTA DE CASTILLA Y LEÓN. Trabajando con los procesos: Guía para la gestión por procesos. Comunidad de Castilla y León: Consejería de Presidencia y Administración Territorial. 2004. ISBN 978-84-9718-264-5.

KAPLAN, R. y NORTON, D. Creating the office of strategy management. Harvard Business Review, 2005, 83(10), 64-74.

LÓPEZ, M.R., SÁNCHEZ, C.P. y MONELOS, P. de L. Mapa de Riesgos: Identificación y Gestión de Riesgos. Atlantic Review of Economics [en línea]. 2013, 2(1). [Consulta: 4 abril 2017]. Disponible en: http://www.unagaliciamoderna.com/eawp/coldata/upload/mapa de riesgos $1906 \quad 06$ 13.pdf.

LYON, B.K. y HOLLCROFT, B. Evaluaciones de riesgos: Las 10 deficiencias principales y consejos para mejorar. Professional Safety [en línea]. 2012, 28-34. [Consulta: 30 enero 2018]. Disponible en: http://www.asse.org/assets/1/7/028 034 F1Lyon 12122.pdf.

MALLETTE, P. y HOPKINS, W.E. Structural and cognitive antecedents to middle management influence on strategic inertia. Journal of Global Business Management [en línea]. 2013, 9(3), 104-115. [Consulta: 8 febrero 2018]. Disponible en: https://search.proquest.com/openview/09 $\underline{\text { b670899a8f9b729303784a6e784d8b/1?pq-origsite }=g s c h o l a r \& c b l=406316}$

STACHUK, M.J.M., PÉREZ, G.H. y JUSCZYSCZYN, J.C.M. Gestión estratégica de recursos tecnológicos en pequeños aserraderos. Ingeniería Industrial [en línea]. 2013, 34(3), 328339. [Consulta: 27 marzo 2018]. Disponible en: http://scielo.sld.cu/scielo.php?script=sci abstract\&pid=S1815-59362013000300009\&lng=es\&nrm=iso\&tlng=es.

MARTIN, G.P., MEJIA, L.R.G. y WISEMAN, R.M. Executive Stock Options as Mixed Gambles: Revisiting the Behavioral Agency Model. Academy of Management Journal [en línea]. 2013, 56(2), 451-472. [Consulta: 30 enero 2018]. Disponible en: http://amj.aom.org/cgi/doi/10.5465/ amj.2010.0967.

MELO, J.C. Gestión de riesgos en la Organización: Teoría y práctica. La Habana, Cuba: Editorial Academia. 2015. ISBN 978-959-270-335-3.

MITCHELL, R., OBEIDAT, S. y BRAY, M. The Effect of Strategic Human Resource Management on Organizational Performance: The Mediating Role of High-Performance Human Resource Practices. Human Resource Management [en línea]. 2013, 52(6), 899-921. [Consulta: 30 enero 2018]. Disponible en: http://doi.wiley.com/10.1002/hrm.21587.

MOYA, J.V., VILLACRES, M.B.J. y LEÓN, A.M. Modelo conceptual para la planificación estratégica con la incorporación de la responsabilidad social universitaria. Ingeniería Industrial, 2013, 34(1), 77-86.

MURILLO, L. Premisas para la Planeación. [en línea], 2012. Disponible en: http://es.slideshare. net/hunter_lili/premisas-para-la-planeacion. 
NEGRÍN, E. Metodología para el perfeccionamiento de los procesos en empresas hoteleras. Revista del Centro de Investigación de Ciencias Administrativas y Gerenciales [en línea]. 2003, 2(2). Disponible en: http://publicaciones.urbe.edu/index.php/cicag/article/view/547/1346.

ORNÉS, S. Gestión Integral de Riesgo: rol del municipio venezolano desde lo normativo. Revista Multiciencias [en línea]. 2011, 11(2), 160-165. [Consulta: 4 junio 2017]. Disponible en: http://produccioncientificaluz.org/index.php/multiciencias/article/view/16849/16823.

RAMÍREZ, L.M. Actualizaciones para el Desarrollo Organizacional. S.I.: Juan Carlos Martínez Coll. 2005. ISBN 978-84-689-6125-5.

ROMÁN, M.E. Ámbito Empresarial: la Gestión por Procesos. Su implementación e importancia en la práctica empresarial. Revista informativa del Centro CIMEX de Capacitación, 2006, 9(3), 28-31.

RUMMLER, G.A. y BRACHE, A.P. Improving performance: how to manage the white space on the organization chart. S.I.: Jossey-Bass. 1995. ISBN 978-1-118-14370-4.

SÁNCHEZ, M.M. Gestión del cambio y Planificación Estratégica: En instituciones de Educación Superior. Palermo Business Review, 2016, 13, 51-72.

VALDÉS, A.E., SÁNCHEZ, R.S. y MARTÍNEZ, A.F.A. Procedimiento para el control de gestión en la Empresa Campismo Popular de Villa Clara. Ingeniería Industrial [en línea]. 2013, 34(2), 198-211. [Consulta: 27 marzo 2017]. Disponible en: http://scielo.sld.cu/pdf/rii/v34n2/rii09213. pdf.

VALLEJO, L.M.P., CORELLA, C.M.V. y CAMPAÑA, M.P. Desarrollo de las capacidades dinámicas para la implementación de cambios organizacionales. Revista Ingeniería Industrial. Universidad de Concepción, Concepción, Chile, 2015, 14(3), 81-93.

ZARATIEGUI, J.R. La Gestión por Procesos: su papel e importancia en la empresa. Economía Industrial [en línea]. 1999, 6(330), 81-88. [Consulta: 16 marzo 2015]. Disponible en: https:// www.virtuniversidad.com/greenstone/collect/administracion/import/Cuatrimestre\%20X/ An\%C3\%A1lisis\%20del\%20Entorno\%20y\%20Estrategia\%20Administrativa\%20 Empresarial/gesti\%C3\%B3nporprocesos.pdf. 